\title{
Novel anti-angiogenic effects of formononetin in human colon cancer cells and tumor xenograft
}

\author{
KATHY KA-WAI AUYEUNG, PUI-CHING LAW and JOSHUA KA-SHUN KO \\ Center for Cancer and Inflammation Research, School of Chinese Medicine, \\ Hong Kong Baptist University, Hong Kong SAR, P.R. China
}

Received July 2, 2012; Accepted August 17, 2012

DOI: 10.3892/or.2012.2056

\begin{abstract}
Formononetin is a novel herbal isoflavonoid isolated from Astragalus membranaceus, a medicinal plant that possesses antitumorigenic properties. Our previous findings demonstrated that formononetin initiates growth-inhibitory and pro-apoptotic activities in human colon cancer cells. In the present study, we aimed to further examine the potential of formononetin in controlling angiogenesis and tumor cell invasiveness in human colon cancer cells and tumor xenografts. The results showed that formononetin downregulated the expression of the key pro-angiogenic factors, including vascular endothelial growth factor (VEGF) and matrix metalloproteinases. We also discovered that the invasiveness of metastatic colon cancer cells was alleviated following drug treatment. The potential anti-angiogenic effect of formononetin was examined in nude mouse xenografts. The tumor size and the number of proliferating cells were reduced in the tumor tissues obtained from the formononetin-treated group. The serum VEGF level was also reduced in the drug-treated animals when compared to the controls. These findings suggest that formononetin inhibits angiogenesis and tumor cell invasion, and thus support its use in the treatment of advanced and metastatic colon cancers.
\end{abstract}

\section{Introduction}

Angiogenesis is essential in the process of carcinogenesis to facilitate tumor progression and metastasis. The vascular endothelial growth factor (VEGF) is a well characterized angiogenic factor known to stimulate angiogenesis within a tumor (1). Additionally, the expression and activity of matrix metalloproteinases (MMPs) are increased in almost every type of human cancer, which may be associated with cell invasion, metastasis and angiogenesis. Among the MMPs, MMP-2 and

Correspondence to: Dr Joshua Ka-Shun Ko, Center for Cancer and Inflammation Research, School of Chinese Medicine, Hong Kong Baptist University, 7 Baptist University Road, Hong Kong SAR, P.R. China

E-mail: jksko@hkbu.edu.hk

Key words: formononetin, angiogenesis, cell invasiveness, cell proliferation, colon cancer
MMP-9 directly regulate angiogenesis (2). Another important mechanism for the progression of colorectal cancer is the ability of the transformed cells to invade and traverse the basement membrane, which in turn facilitates the conversion of adenomas into carcinomas and then metastases (1). MMPs have been implicated in these processes and a previous study has suggested that the plasma level of MMP-2 and MMP-9 may be viewed as a non-invasive indicator of invasiveness and metastasis in colon cancer (3).

The dried root of Astragalus membranaceus (Radix Astragali) has a long history of medicinal use in traditional Chinese medicine as an immunomodulating agent in mixed herbal decoctions to treat the common cold, diarrhea, fatigue and anorexia (4). In contemporary pharmacotherapy, Radix Astragali has been used to ameliorate the side-effects of cytotoxic antineoplastic drugs (5). Among its different constituents, Astragalus polysaccharides have been most widely studied particularly for their immunopotentiating properties, such as stimulation of murine B cell proliferation and cytokine production (6). Apart from these, the results from clinical studies have shown that Astragalus polysaccharides counteract against the side-effects of chemotherapeutic drugs, including a significant attenuation of myelosuppression in cancer patients (7). Nevertheless, our laboratory findings did not support any direct anticancer activity by Astragalus polysaccharides. In recent years, we focused on investigating the anti-carcinogenic properties of total Astragalus saponins (AST) in human colon cancer cells and tumor xenografts. Our results indicated that AST inhibits cell proliferation through phase-specific cell cycle arrest and caspase-dependent apoptosis. The reduction in tumor volume as well as pro-apoptotic and anti-proliferative effects in HT-29 nude mice xenograft are comparable to those produced by 5-FU. In addition, the side-effects associated with the drug combo 5-FU and oxaliplatin may be alleviated by AST (8). These data indicate that AST may be an effective chemotherapeutic agent in colon cancer treatment, and may also be used as an adjuvant in combination with other orthodox chemotherapeutic drugs to reduce the side-effects of the latter compounds. Subsequent study using multiple human cancer cell lines, including those of the colon, stomach and liver, further indicated the universal growth-inhibitory and pro-apoptotic effects of AST. We have also confirmed that non-steroidal antiinflammatory drug activated gene (NAG-1) is a major molecular target of AST during its antitumorigenic action, which has a 
strong correlation with the PI3K-Akt signaling pathway during its action (9). Among various active constituents, information regarding the bioactivity and anticarcinogenic potential of Astragalus flavonoids remains inadequate.

Formononetin is one of the major isoflavonoid constituents isolated from Astragalus membranaceus in sufficient quantity with $>95 \%$ purity (10). Apart from Astragalus, formononetin can also be found in other commonly used herbs in China, Japan and the Western world, including the root of Glycyrrhiza glabra and Glycyrrhiza uralensis (licorice) (11). Formononetin has demonstrated diverse pharmacological benefits. As a phytoestrogen, it has demonstrated a metabolic effect by upregulating interleukin-4 production in activated T cells via increased AP-1 DNA binding activity (12). It also possesses anti-inflammatory activity by significantly suppressing arachidonic acid release in HT-29 human colon cancer cells (13). Formononetin also reduces blood pressure and central arterial stiffness, leading to reduced cardiovascular risk (14). Furthermore, its value as a potential antidiabetic agent in regulating lipid metabolism by the activation of PPAR- $\alpha$ and PPAR- $\gamma$ receptors has been indicated (10). Formononetin has demonstrated analgesic activity in an acetic acid-induced abdominal constriction mouse model (15). Nevertheless, this novel compound has also been shown to induce the proliferation of osteoblasts (16). We recently reported that formononetin, similar to AST, can induce growth inhibition and promote caspase-dependent apoptosis in human colon cancer cells. This involves the inhibition of antiapoptotic proteins, such as $\mathrm{Bcl}-2$ and $\mathrm{Bcl}-\mathrm{x}_{\mathrm{L}}$ and the activation of the novel pro-apoptotic protein, NAG-1 (17). However, data on the influence of formononetin on tumor angiogenesis are insufficient. In the present study, we extended our ongoing study by investigating the effects of formononetin on angiogenesis and the invasiveness of human colon cancer cells and nude mouse xenografts. The results obtained provide evidence for the broader use of formononetin as a chemotherapeutic agent against advanced and metastatic human colorectal cancers, apart from its use a pro-apoptotic agent.

\section{Materials and methods}

Drugs and chemicals. The structure of formononetin is illustrated in Fig. 1A. The drug chemical used in this study had the purity of $>99 \%$ and was purchased from Fluka (Buchs, Switzerland). The types and sources of antibodies used were as follows: anti-proliferating cell nuclear antigen (PCNA), anti-VEGF, anti-MMP-2 and anti-MMP-9 (Santa Cruz Biotechnology, Santa Cruz, CA); and anti- $\beta$-actin (SigmaAldrich, St. Louis, MO). The BioCoat Matrigel Cell Invasion Chamber was purchased from BD Biosciences (San Jose, CA). All other chemicals were obtained from Sigma-Aldrich unless specified.

Cell culture. HCT-116 human colorectal carcinoma cells (CCL-247) and LoVo human colorectal adenocarcinoma cells from metastatic sites (CCL-229) were purchased from ATCC (Manassas, VA). HCT-116 and LoVo cells were grown in $75-\mathrm{cm}^{2}$ flasks and incubated at $37^{\circ} \mathrm{C}$ under a $5 \% \mathrm{CO}_{2}$ atmosphere in DMEM-1640 medium (Gibco, Carlsbad, CA) and F-12K Medium (ATCC), respectively supplemented with $10 \%$ FBS (Gibco) plus $1 \%$ penicillin and streptomycin (Gibco). LoVo cells were chosen for the invasion test due to their metastatic properties.

Tumor xenografts in nude mice and drug treatment. The experimental procedures were approved by our institutional animal research ethics committee with reference to the European Community guidelines for the use of experimental animals. Five-week-old female Balb/c-nu/nu mice were obtained from the Laboratory Animal Service Unit of the Chinese University of Hong Kong. The animals were kept under sterile conditions and in isolated pathogen-free ventilation chambers under an ambient temperature of $20-22^{\circ} \mathrm{C}$ and $40-50 \%$ relative humidity. The mice were anesthetized and injected subcutaneously near the flank with HCT-116 cells as previously described (8). Ten days after tumor induction, the mice began to receive either DMSO (control) or formononetin $(20 \mathrm{mg} / \mathrm{kg})$ treatment by daily intraperitoneal injection for 14 days. Tumor volume (in $\mathrm{mm}^{3}$ ) was measured every other day (8), while tumor mass (in grams) was calculated on the final day of the experiment. The animals were sacrificed on day 24, with their serum collected and tumor tissues excised. Apart from the mainstream experiments, we also obtained whole blood from normal Balb/c mice to measure the total white blood cell (WBC) count. This could allow us to assess the influence of formononetin on the immune system of experimental animals. Blood was collected from the tail vein of mice at the end of the experimental period. The sampled blood $(20 \mu \mathrm{l})$ was mixed with $176 \mu \mathrm{l}$ of $3 \%$ acetic acid and $4 \mu \mathrm{l}$ of $1 \%$ crystal violet. Stained WBCs were counted in a hemocytometer under a light microscope (magnification, $\mathrm{x} 200$ ). The body weight of all the animals was recorded throughout the entire experimental period as an assessment of drug toxicity. Any mortality during the course of the study was recorded.

Immunohistochemical analysis of cell proliferation and VEGF in tumor tissues. The excised tumor samples were fixed for paraffin embedment and tumor sections $(5 \mu \mathrm{m})$ were prepared on slides. Immunohistochemical analysis for cell proliferation and VEGF expression was performed. The sections were blocked with $2 \%$ BSA to minimize non-specific binding, and incubated overnight with anti-PCNA or anti-VEGF monoclonal antibody at $4^{\circ} \mathrm{C}$. The positively stained cells were visualized by incubating the sections with 3,3'-diaminobenzidine using Mayer's hematoxylin as the counterstain. The negative control was prepared with similar procedures but without the addition of the primary antibody.

Western immunoblotting. The cells (treated for 0-72 h with DMSO or $200 \mu \mathrm{M}$ formononetin) were lysed in RIPA buffer containing $50 \mathrm{mM}$ Tris, $150 \mathrm{mM} \mathrm{NaCl}, 0.5 \%$ sodium deoxycholate, $0.1 \%$ SDS, 2 mM EDTA, $0.1 \%$ Triton $\mathrm{X}-100$, $10 \%$ glycerol, $1 \mathrm{mM}$ phenylmethylsulfonyl fluoride, $10 \mu \mathrm{g} / \mathrm{ml}$ aprotinin and $5 \mu \mathrm{g} / \mathrm{ml}$ pepstatin A. After the insoluble materials were removed by centrifugation at $14,000 \mathrm{x}$ g for $10 \mathrm{~min}$ at $4^{\circ} \mathrm{C}$, the proteins were quantified using the Coomassie plus Protein Assay Reagent kit (Pierce, Rockford, IL). Total cellular proteins (20-40 $\mu \mathrm{g}$ ) in the cell lysates were electrophoresed and transferred onto nitrocellulose membranes for western immunoblotting as previously described (9). The results were analyzed using Quantity One version 4.4.1 Basic software (Bio-Rad Laboratories, Hercules, CA). 


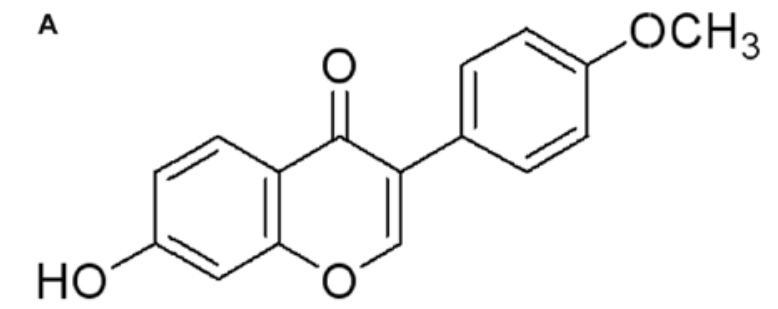

B
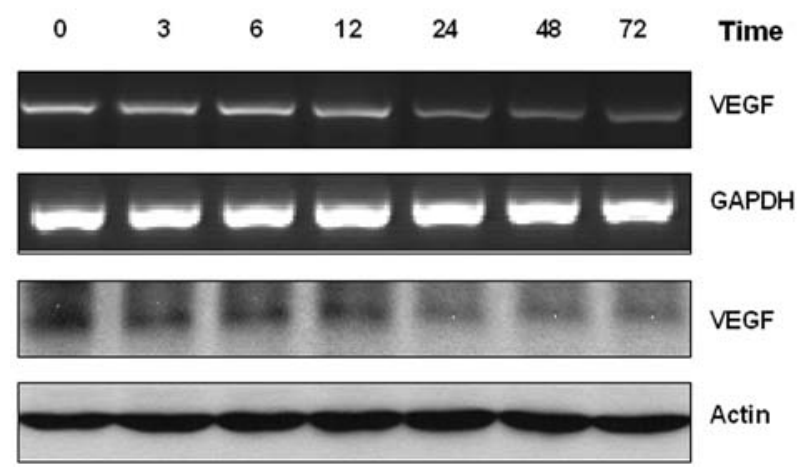

c
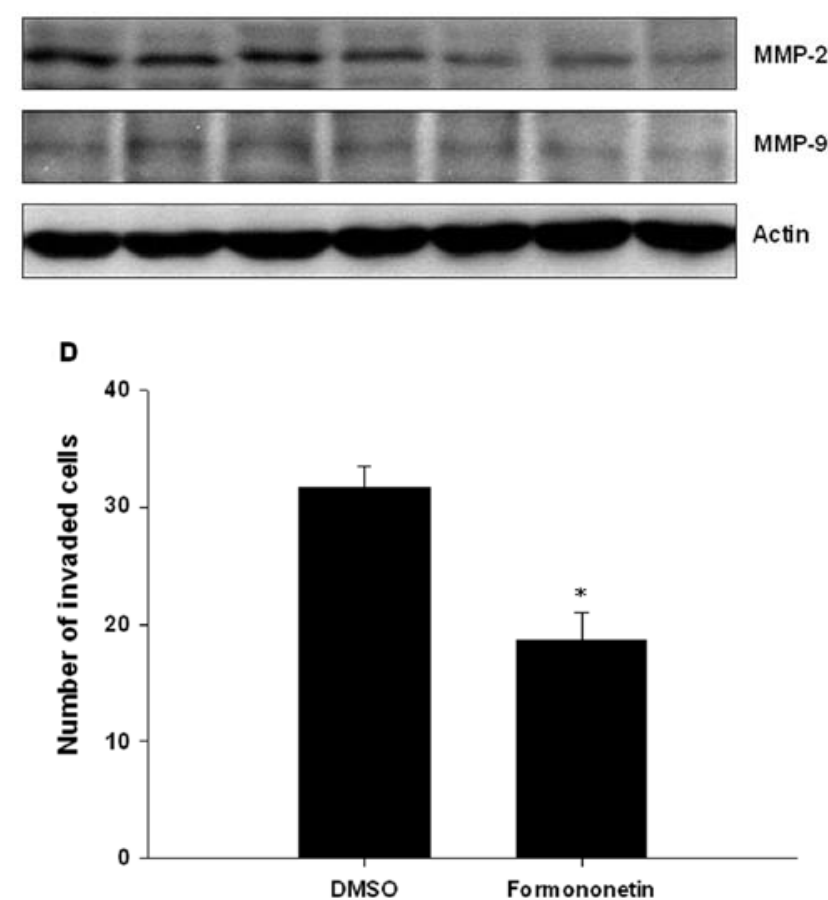

Figure 1. Anti-angiogenic and anti-invasive effects of formononetin in colon cancer cells. (A) Chemical structure of formononetin (7-hydroxy-4'-methoxyisoflavone). HCT-116 cells were treated with formononetin $(200 \mu \mathrm{M})$ for various periods of time $(0-72 \mathrm{~h})$. The $(\mathrm{B})$ gene and protein expression of VEGF, as well as the (C) protein expression of MMP-2 and MMP-9 in the formononetin-treated cells were determined by real-time RT-PCR and western immunoblotting. Representative protein bands were obtained from 3 separate experiments performed in duplicate. The intensity of the target bands was normalized to $\beta$-actin re-probed on the same immunoblot. (D) The effect of formononetin on LoVo cell invasiveness was examined using the BioCoat Matrigel Invasion Chamber. Data are summarized and values are expressed as the means \pm SEM of 3-5 tumor tissue sections. ${ }^{*} \mathrm{P}<0.05$, significantly different from the DMSO control.

Real-time reverse transcriptase-PCR (RT-PCR). The cells were lysed in TRIzol ${ }^{\circledR}$ reagent to extract total cellular RNA.
Single-stranded cDNA was synthesized from $5 \mu \mathrm{g}$ of total RNA using M-MLV reverse transcriptase. The sequences of VEGF (NM_001025370, 226 bp) used for PCR were: 5'-AAG GAGGAGGGCAGAATCAT-3' (forward) and 5'-ATCTGCA TGGTGATGTTGGA-3' (reverse). Those for GAPDH (595 bp) were used as the internal control: 5'-CCACCCATGGCAAA TTCCATGGCA-3' (forward) and 5'-TCTAGACGGCAGGTC AGGTCCACC-3' (reverse). The conditions for PCR amplification (GeneAmp PCR System 9700, Applied Biosystems, Foster City, CA) were established as follows: 30 cycles of $95^{\circ} \mathrm{C}$ for $2 \mathrm{~min}, 94^{\circ} \mathrm{C}$ for $30 \mathrm{sec}, 60^{\circ} \mathrm{C}$ for $30 \mathrm{sec}$ and $72^{\circ} \mathrm{C}$ for $30 \mathrm{sec}$, followed by an additional 10 -min extension at $72^{\circ} \mathrm{C}$. The RNA products were resolved by electrophoresis on a $1.8 \% \mathrm{TAE}$ agarose gel stained with ethidium bromide. All the reagents used in real-time RT-PCR were purchased from Invitrogen (Carlsbad, CA).

Cell invasion assay. The invasive ability of LoVo cells was assessed using a BioCoat Matrigel Invasion Chamber (BD Falcon) as previously described (18). In brief, cells were trypsinized and suspended in serum-free culture medium at the concentration of $1 \times 10^{6} / \mathrm{ml}$ in the upper chamber. The lower chamber was filled with culture medium supplemented with $10 \% \mathrm{FBS}$ as the chemoattractant. After $24 \mathrm{~h}$ of drug treatment (200 $\mu \mathrm{M}$ formononetin, or DMSO as the control), the invaded cells that had passed through Matrigel and had adhered to the lower surface of the membrane were fixed with methanol and stained with $0.1 \%$ crystal violet. The stained cells were viewed and counted under a microscope (x200 magnification).

Serum VEGF level. The serum VEGF level in tumor xenografted nude mice was determined using a VEGF immunoassay kit (Invitrogen). The procedures for the measurement of the serum VEGF level was carried out according to the protocols listed in the manufacturer's manual.

Statistical analysis. Numerical data are expressed as the means \pm standard error of the mean (SEM). Statistical significance of at least $\mathrm{P}<0.05$ was determined by one-way analysis of variance (ANOVA) followed by a post-hoc least significant sifference (LSD) test using SPSS version 10.0 software (SPSS, Chicago, IL).

\section{Results}

Effects of formononetin on VEGF and MMP expression in human colon cancer cells. In order to determine whether formononetin exerts anti-angiogenic effects in human colon cancer cells, the protein expression of bFGF, VEGF and MMPs was assessed. Both the gene and protein expression of VEGF were downregulated in the formononetin-treated HCT-116 cells, indicating the suppression of angiogenesis (Fig. 1B). MMP-2 and MMP-9 expression was also investigated as their activation contributes to tumor invasion and metastasis. Our results demonstrated that formononetin inhibited the protein expression of MMP-2 and MMP-9 (Fig. 1C). The formononetin-induced downregulation of pro-angiogenic factors occurred in a time-dependent manner, being more pronounced after $24 \mathrm{~h}$ of drug treatment. We then examined the effect of formononetin on tumor cell invasiveness using the 
A
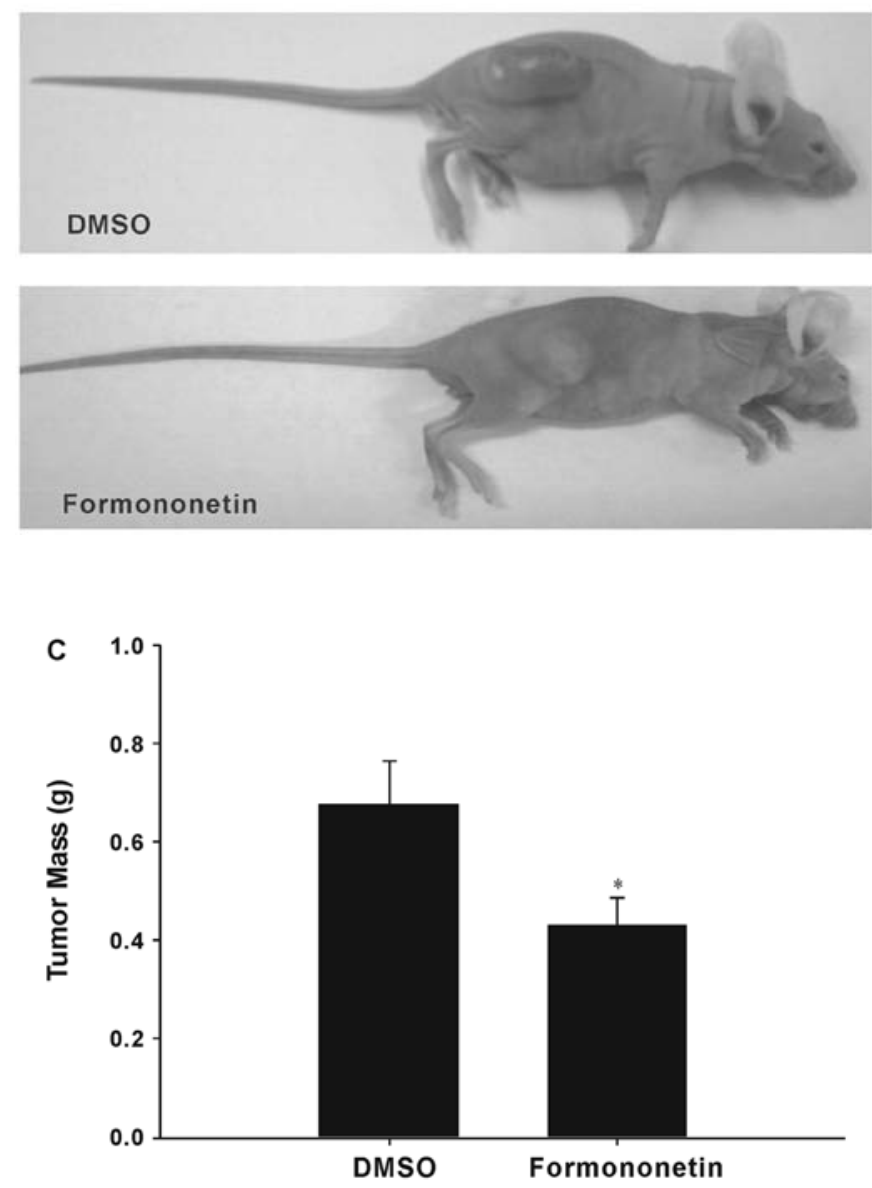

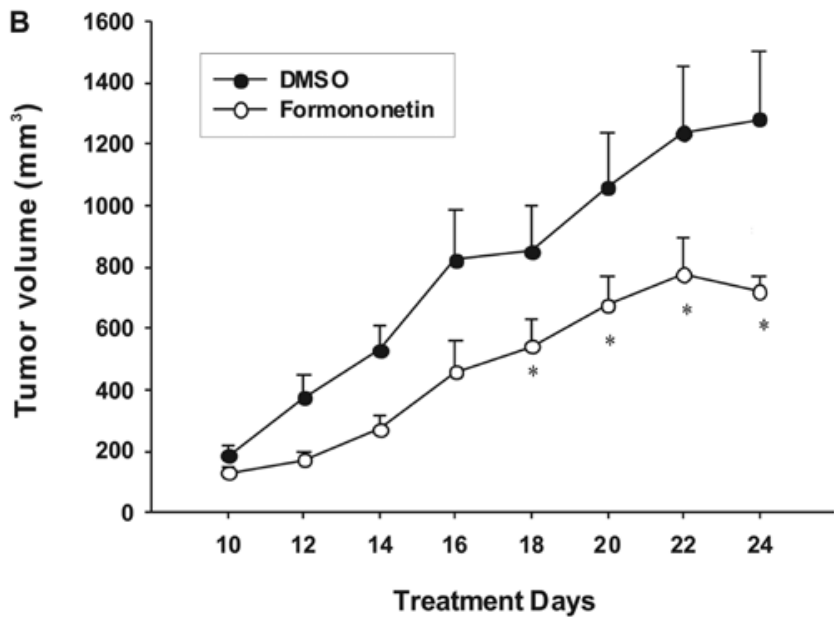

D



Figure 2. In vivo antitumor effects of formononetin. (A) Images showing the HCT-116 xenografted nude mice in th 2 treatment groups. The upper panel indicates mice treated with DMSO (control) and the lower panel indicates the mice treated with $20 \mathrm{mg} / \mathrm{kg}$ of formononetin, both by intraperitoneal injection once daily for 2 weeks. Tumor volume (B; in $\mathrm{mm}^{3}$ ) and tumor mass $(\mathrm{C}$; in grams) were recorded in the nude mouse xenografts. (D) The white blood cell (WBC) count in the formononetin-treated mice was also determined. Values are expressed as the means \pm SEM ( $\mathrm{n}=6-8$ animals). ${ }^{*} \mathrm{P}<0.05$, significantly different from the DMSO control.

metastatic colon cancer cell line, LoVo. The number of cells that had passed through the Matrigel membrane was counted in both control and formononetin-treated groups. The data are summarized in Fig. 1D. The average number of invaded cells in the formononetin-treated group was significantly decreased when compared with the DMSO control group. Based on these findings, we suggest that the anticarcinogenic effects of formononetin involve the inhibition of angiogenesis and tumor cell invasiveness.

Formononetin inhibits tumor growth in nude mouse xenografts. In order to explore the antitumorigenic effect of formononetin against colon cancer development in vivo, HCT-116 nude mouse xenografts were used. No mortality or significant change in body weight was observed throughout the experimental period in both the control- and drug-treated animals. Fig. 2A shows the appearance and relative size of the tumors in the control and formononetin-treated animals. On the final day of the experiment, tumor tissue samples were obtained and the tumor volume in the formononetin-treated group was found to be reduced by $44 \%\left(1280 \pm 222 \mathrm{~mm}^{3}\right.$ in the DMSO-treated control vs. $718.6 \pm 52.6 \mathrm{~mm}^{3}$ in the formononetin-treated group)
(Fig. 2B). Tumor mass was also decreased by approximately $37 \%$ (Fig. 2C). Our results also showed that the WBC counts were comparable between the DMSO- and formononetintreated groups (Fig. 2D), indicating that formononetin exerted no neutropenic effect on the experimental animals, as in the case of some conventional chemotherapeutic drugs.

Formononetin reduces VEGF serum levels and tumor tissue expression and inhibits tumor cell proliferation. In order to further confirm the effects of formononetin on angiogenesis, serum VEGF levels in the nude mice were measured on the final day of the experiment after the animals were sacrificed. The results showed that formononetin markedly reduced the serum VEGF levels in the nude mice by almost $50 \%$ when compared to the control group (Fig. 3A). The excised tumor tissues were then stained for VEGF expression, which showed a reduction in the tissues from the formononetin-treated group when compared with those from the control group (Fig. 3B). In order to determine whether the inhibition of tumor growth was associated with the anti-proliferative effects, an immunohistochemical assessment of PCNA in the tumor samples was also conducted. The results showed that formononetin treat- 


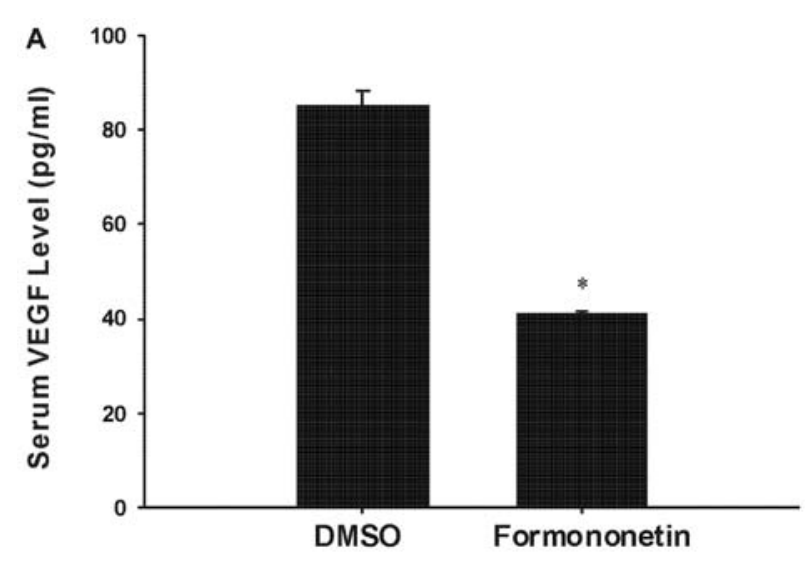

B
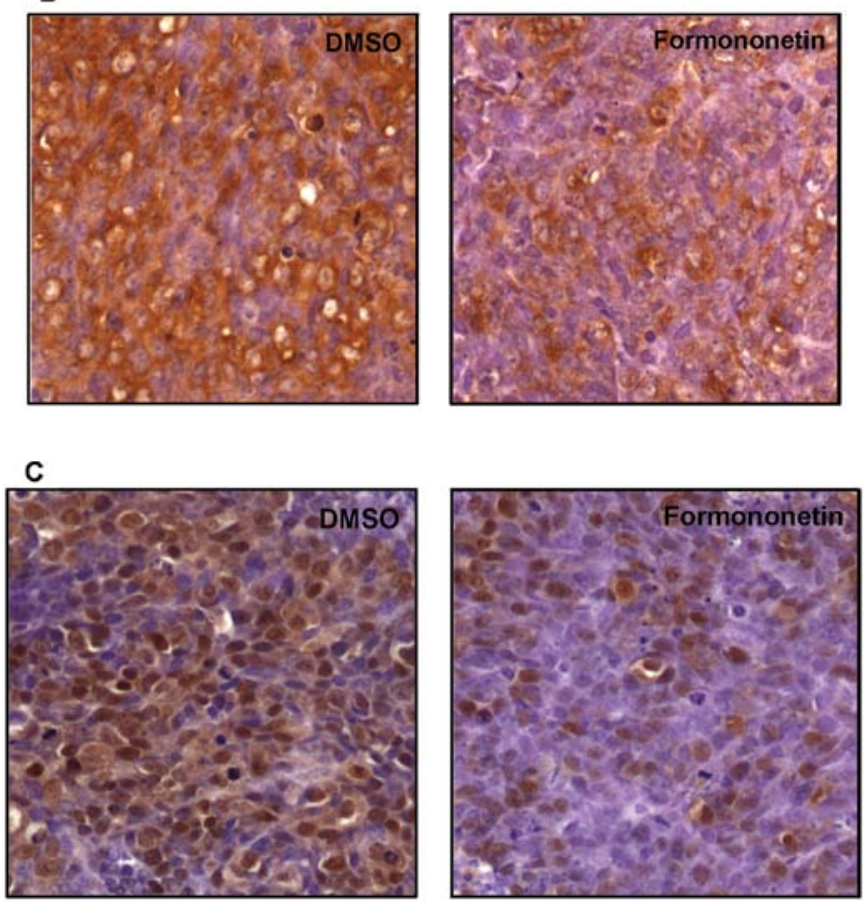

Figure 3. Anti-angiogenic and anti-proliferative effects of formononetin in the tumor xenografts. Determination of serum VEGF levels and immunohistochemical assessment of VEGF expression and cell proliferation (PCNA) in tumor tissue sections were performed. Total serum was collected from the nude mice on the day of sacrifice. (A) Tumor tissue VEGF level was measured using an ELISA kit as described in Materials and methods. Values represent the means \pm SEM of 3 independent experiments. ${ }^{*} \mathrm{P}<0.05$, statistical significance compared to the DMSO control. (B) VEGF- and (C) PCNA-positive cells were visualized in tumor sections excised from the control- (DMSOtreated) or formononetin-treated nude mice. Representative images from 3-5 sections of the control- or formononetin-treated animals are shown.

ment markedly reduced the number of visible PCNA-labeled cells (Fig. 3C), which suggests that formononetin also acts by inhibiting cell proliferation.

\section{Discussion}

Ourongoing studies have indicated that total ASTexerts powerful anticarcinogenic activities against both colon and liver cancer development through target-specific mechanisms $(8,9,19)$. In spite of this, only a few reports (in Chinese) have demonstrated the anti-oxidative properties of Astragalus flavonoids by inhibiting membrane lipid peroxidation. Formononetin, being a major isoflavone constituent of Radix Astragali, possesses anti-inflammatory properties (20), and has also been proposed to be potentially anticarcinogenic (13). We recently showed that formononetin is a strong pro-apoptotic agent in colon cancer cells (17). Nevertheless, formononetin does not appear to exert any direct effect on the cell cycle. This suggests that the proapoptotic effect exhibited by formononetin may not directly correlate with the modulation of mitotic inhibition. In addition to formononetin, we also investigated the potential apoptotic effect exhibited by the total isoflavonoid extract (AFT) (data not shown). We first examined the inhibitory effect exhibited by AFT on colon cancer cell growth and observed that there was a significant decrease in the percentage of viable cells with increasing doses and treatment time. By studying the protein expression of PARP, a key marker of apoptosis, it was determined that AFT exerts a pro-apoptotic effect on colon cancer cells. Through qualitative HPLC analysis, we confirmed the presence of formononetin in AFT as one of the major peaks on the chromatogram. This suggests that the anticarcinogenic activity of AFT may be largely due to formononetin. However, further study is required to determine the exact contribution of formononetin in the anticancer action of AFT.

It is generally known that VEGF facilitates angiogenic activities, while the increased expression of these growth factors has been shown to have a strong correlation with tumor development $(21,22)$. Additionally, cancer cells and tumor stromal cells are involved in the secretion of VEGF in the tumor microenvironment (23). Previous studies have demonstrated that isoflavones help prevent osteoporosis and related menopausal disorders, and reduce cardiovascular risk. Their possible effects on chemoprevention and anti-oxidation have also been demonstrated $(24,25)$. The plant-derived isoflavones, biochanin A and formononetin, are the methylated precursors of genistein and daidzein, respectively. Genistein inhibits endothelial cell proliferation and angiogenesis in vitro (26). It has also found that biochanin $\mathrm{A}$ and genistein inhibit the proliferation of gastric, esophageal and colon cancer cells (27). Daidzein has been shown to prevent osteoporosis and has exhibited anticancer activities on myeloid leukemia, breast cancer and pediatric tumors in vitro (28). Nevertheless, little is known about the anti-angiogenic effect of formononetin on colon cancer. Our current findings indicate that formononetin downregulates both the gene and protein expression of VEGF, implicating its involvement in the process of modulating angiogenesis. Tumor angiogenesis is an essential step in cancer growth and metastasis, and thus it is an important therapeutic target being extensively studied over the years (29). In tumors, neovascularization is necessary for both primary and metastatic tumor growth due to the increase in nutrient consumption. The process of angiogenesis involves the separation of endothelial cells from pericytes and the basement membrane upon the action of proteases, such as MMPs, urokinase plasminogen activators (uPAs) and cathepsins. Invasion and migration across basement membranes and subsequent extension into the tumor mass are triggered by the angiogenic growth factors, such as VEGF, basic fibroblast growth factor (bFGF), transforming growth factor- $\beta$ (TGF- $\beta$ ) and platelet-derived growth factor (PDGF) (30-32). The transition from the avasculature state into a highly angiogenic state (angiogenic switch) is due to the 
imbalance between pro-angiogenic signals and anti-angiogenic signals released by tumor cells and host cells. When pro-angiogenic signals dominate, tumors become vascularized (29). Neovascularization in tumors plays a critical role in colorectal cancer progression, and previous studies have shown that the increase in angiogenesis in the primary tumor correlates with poor prognosis and relapse of the disease $(33,34)$.

In colon cancer, tumor cells must invade through the muscularis mucosa and migrate into the submucosa before they can reach the lymphatic channels or blood vessels. This is an essential process for metastasis to occur (35). An increase in invasion may be related to an increase in cell motility (36) and the secretion of basement membrane-degrading enzymes (e.g., MMPs), which together degrade the extracellular matrix and the basement membrane $(36,37)$. The mechanical pressure generated by the rapidly growing neoplasm eventually pushes tumor cells along tissue planes with minimal resistance (38). In this study, we demonstrate that formononetin reduces the protein expression of MMP-2 and MMP-9 and suppresses tumor cell invasiveness. These MMPs have been proposed to serve as a marker of colorectal cancer development (3). In fact, a novel antisense-based inhibitor of MMP-9 has been found to be capable of attenuating angiogenesis as well as human prostate cancer cell invasion, leading to the suppression of tumorigenicity (39). MMP-2 (also known as gelatinase A) is localized at specific surface protrusions on the cell membrane and promotes invasion (40), whereas MMP-9 (also known as gelatinase B) causes key processes in tumor cell migration, invasion, angiogenesis and tumor growth $(41,42)$. The expression of MMP-9 in tumor epithelial cells positively correlates with colorectal cancer progression due to its control over essential functions mediating metastasis (43). Other studies have indicated that MMP-9 secreted by colon cancer cells promotes extracellular matrix degradation, and assists tumor cell seeding (44). The facilitation of tumor invasion and neovascularization by MMP-9 is mainly caused by the cleavage of specific factors, such as collagen IV in the extracellular matrix (45). Hence, targeting MMP-9 in tumor epithelial cells is regarded as a key therapeutic strategy for controlling the progression of colorectal cancer (46). MMP-9 is not usually expressed in normal human tissues but is mainly found in invasive tumors. It has been reported that the inhibition of MMP-9 expression leads to ERK-mediated p16 expression, resulting in cell cycle arrest (47) while at the same time inducing apoptosis (48). This suggests that the impact of MMP-9 inhibition is not restricted to the suppression of tumor angiogenesis and invasiveness, but also contributes to the promotion of apoptosis. This proposition coincides with our findings on the pro-apoptotic properties of formononetin, although cell cycle arrest was not directly facilitated.

Our present study provides evidence that formononetin controls colon tumor progression without causing significant toxicity in drug-treated animals, with no mortality or drop in body weight. This observation was confirmed by the fact that formononetin did not exert any neutropenic effect on the drugtreated animals. Therefore, we can conclude that formononetin is capable of inducing tumor growth inhibition as well as antiangiogenic and anti-invasive effects with minimal adverse drug reactions. These important findings suggest that formononetin could be further developed as a potential chemotherapeutic/ adjuvant agent in the treatment of colon cancers, including the advanced and metastatic types.

\section{Acknowledgements}

This study was supported by the HKBU Faculty Research Grant (FRG/07-08/II-02).

\section{References}

1. Boedefeld WM, Bland KI and Heslin MJ: Recent insights into angiogenesis, apoptosis, invasion, and metastasis in colorectal carcinoma. Ann Surg Oncol 10: 839-851, 2003.

2. Egeblad M and Werb Z: New functions for the matrix metalloproteinases in cancer progression. Nat Rev Cancer 2: 161-174, 2002.

3. Tutton MG, George ML, Eccles SA, Burton S, Swift RI and Abulafi AM: Use of plasma MMP-2 and MMP-9 levels as a surrogate for tumour expression in colorectal cancer patients. Int J Cancer 107: 541-550, 2003.

4. Bensky D, Gamble A and Kaptchuk TK: Chinese Herbal Medicine Materia Medica. Eastland Press, Seattle, pp358-359, 1993.

5. Zee-Cheng RK: Shi-quan-da-bu-tang (ten significant tonic decoction), SQT. A potent Chinese biological response modifier in cancer immunotherapy, potentiation and detoxification of anticancer drugs. Methods Find Exp Clin Pharmacol 14: 725-736, 1992.

6. Shao BM, Xu W, Dai H, Tu P, Li Z and Gao XM: A study on the immune receptors for polysaccharides from the roots of Astragalus membranaceus, a Chinese medicinal herb. Biochem Biochem Biophys Res Commun 320: 1103-1111, 2004.

7. Duan P and Wang ZM: Clinical study on effect of Astragalus in efficacy enhancing and toxicity reducing of chemotherapy in patients of malignant tumor. Zhongguo Zhong Xi Yi Jie He Za Zhi 22: 515-517, 2002 (In Chinese).

8. Tin MM, Cho CH, Chan K, James AE and Ko JK: Astragalus saponins induce growth inhibition and apoptosis in human colon cancer cells and tumor xenograft. Carcinogenesis 28: 1347-1355, 2007.

9. Auyeung KK, Cho $\mathrm{CH}$ and Ko JK: A novel anticancer effect of Astragalus saponins: transcriptional activation of NSAIDactivated gene. Int J Cancer 125: 1082-1091, 2009.

10. Shen P, Liu MH, Ng TY, Chan YH and Yong EL: Differential effects of isoflavones, from Astragalus membranaceus and Pueraria thomsonii, on the activation of PPARalpha, PPARgamma, and adipocyte differentiation in vitro. J Nutr 136: 899-905, 2006.

11. Vaya J, Belinky PA and Aviram M: Antioxidant constituents from licorice roots: isolation, structure elucidation and antioxidant capacity toward LDL oxidation. Free Rad Biol Med 23: 302-312, 1997.

12. Park J, Kim SH, Cho D and Kim TS: Formononetin, a phytooestrogen, and its metabolites up-regulate interleukin-4 production in activated T cells via increased AP-1 DNA binding activity. Immunology 116: 71-81, 2005.

13. Jun M, Hong J, Jeong WS and Ho CT: Suppression of arachidonic acid metabolism and nitric oxide formation by kudzu isoflavones in murine macrophages. Mol Nutr Food Res 49: 1154-1159, 2005.

14. Nestel P, Fujii A and Zhang L: An isoflavone metabolite reduces arterial stiffness and blood pressure in overweight men and postmenopausal women. Atherosclerosis 192: 184-189, 2007.

15. Huo Y, Guo C, Zhang QY, Chen WS, Zheng HC, Rahman K and Qin LP: Antinociceptive activity and chemical composition of constituents from Caragana microphylla seeds. Phytomedicine 14: 143-146, 2007.

16. Dong TTX, Zhao KJ, Gao QT, et al: Chemical and biological assessment of a chinese herbal decoction containing Radix Astragali and Radix Angelicae Sinensis: determination of drug ratio in having optimized properties. J Agric Food Chem 54: 2767-2774, 2006.

17. Auyeung KK and Ko JK: Novel herbal flavonoids promote apoptosis but differentially induce cell cycle arrest in human colon cancer cell. Invest New Drugs 28: 1-13, 2010. 
18. Albini A, Iwamoto Y, Kleinman HK, Martin GR, Aaronson SA, Kozlowski JM and McEwan RN: A rapid in vitro assay for quantitating the invasive potential of tumor cells. Cancer Res 47: 3239-3245, 1987.

19. Auyeung KK, Law PC and Ko JK: Astragalus saponins induce apoptosis via an ERK-independent NF- $\kappa \mathrm{B}$ signaling pathway in the human hepatocellular HepG2 cell line. Int J Mol Med 23: 189-196, 2009.

20. Ko JKS, Lam FYL and Cheung APL: Amelioration of experimental colitis by Astragalus membranaceus through antioxidation and inhibition of adhesion molecule synthesis. World $\mathbf{J}$ Gastroenterol 11: 5787-5794, 2005.

21. Kondo Y, Arii S, Mori A, Furutani M, Chiba T and Imamura M: Enhancement of angiogenesis, tumor growth, and metastasis by transfection of vascular endothelial growth factor into LoVo human colon cancer cell line. Clin Cancer Res 6: 622-630, 2000.

22. Sukhthankar M, Yamaguchi K, Lee SH, McEntee MF, Eling TE, Hara Y and Baek SJ: A green tea component suppresses posttranslational expression of basic fibroblast growth factor in colorectal cancer. Gastroenterology 134: 1972-1980, 2008.

23. Carusi D: Phytoestrogens as hormone replacement therapy: an evidence-based approach. Prim Care Update Ob Gyns 7: 253-259, 2000.

24. Liang WC, Wu X, Peale FV, Lee CV, et al: Cross-species vascular endothelial growth factor (VEGF)-blocking antibodies completely inhibit the growth of human tumor xenografts and measure the contribution of stromal VEGF. J Biol Chem 28 951-961, 2006

25. Arjmandi BH: The role of phytoestrogens in the prevention and treatment of osteoporosis in ovarian hormone deficiency. J Am Coll Nutr 20: S398-S402, 2001.

26. Fotsis T, Pepper M, Adlercreutz H, Fleischmann G, Hase T, Montesano R and Schweigerer L: Genistein, a dietary-derived inhibitor of in vitro angiogenesis. Proc Nat Acad Sci USA 90: 2690-2694, 1993.

27. Yanagihara $\mathrm{K}$, Ito $\mathrm{A}$, Toge $\mathrm{T}$ and Numoto $\mathrm{M}$ : Antiproliferative effects of isoflavones on human cancer cell lines established from the gastrointestinal tract. Cancer Res 3: 5815-5821, 1993.

28. Beck V, Rohr U and Jungbauer A: Phytoestrogens derived from red clover: an alternative to estrogen replacement therapy? J Steroid Biochem Mol Biol 94: 499-518, 2005.

29. Harris AL: Angiogenesis as a new target for cancer control. Eur J Cancer Suppl 1: 1-12, 2003

30. Fukumura D, Xavier R and Sugiura T: Tumor induction of VEGF promoter activity in stromal cells. Cell 94: 715-725, 1998.

31. Hanahan D, Christofori G, Naik P and Arbeit J: Transgenic mouse models of tumour angiogenesis: the angiogenic switch, its molecular controls, and prospects for preclinical therapeutic models. Eur J Cancer 32A: 2386-2393, 1996.

32. Hanahan D and Folkman J: Patterns and emerging mechanisms of the angiogenic switch during tumorigenesis. Cell 86: 353-364, 1996.
33. Takebayashi Y, Aklyama S, Yamada K, Akiba S and Aikou T: Angiogenesis as an unfavorable prognostic factor in human colorectal carcinoma. Cancer 78: 226-231, 1996.

34. Vermeulen PB, Van den Eynden GG and Huget P: Prospective study of intratumoral microvessel density, p53 expression and survival in colorectal cancer. Br J Cancer 79: 316-322, 1999.

35. Portera CA Jr, Berman RS and Ellis LM: Molecular determinants of colon cancer metastasis. Surg Oncol 7: 183-195, 1998.

36. Gutman M and Fidler IJ: Biology of human colon cancer metastasis. World J Surg 19: 226-234, 1995.

37. Radinsky R and Ellis LM: Molecular determinants of colon cancer metastasis. Surg Oncol Clin N Am 5: 215-229, 1996.

38. Gabbert $\mathrm{H}$ : Mechanisms of tumor invasion: evidence from in vivo observations. Cancer Metastasis Rev 4: 293-309, 1985.

39. London CA, Sekhon HS, Arora V, Stein DA, Iversen PL and Devi GR: A novel antisense inhibitor of MMP-9 attenuates angiogenesis, human prostate cancer cell invasion and tumorigenicity. Cancer Gene Ther 10: 823-832, 2003.

40. Nakahara H: Transmembrane/cytoplasmic domain-mediated membrane type 1-matrix metalloprotease docking to invadopodia is required for cell invasion. Proc Natl Acad Sci USA 94: 7959-7964, 1997.

41. Coussens LM, Fingleton B and Matrisian LM: Matrix metalloproteinase inhibitors and cancer: trials and tribulations. Science 295: 2387-2392, 2002

42. Fridman R, Toth M, Chvyrkova I, Meroueh SO and Mobashery S: Cell surface association of matrix metalloproteinase-9 (gelatinase B). Cancer Metastasis Rev 22: 153-166, 2003.

43. Zuzga D, Gibbons AV and Li P: Overexpression of matrix metalloproteinase 9 in tumor epithelial cells correlates with colorectal cancer metastasis. Clin Transl Sci 1: 136-141, 2008.

44. Lubbe WJ, Zhou ZY, Fu W, et al: Tumor epithelial cell matrix metalloproteinase- 9 is a target for antimetastatic therapy in colorectal cancer. Clin Cancer Res 12: 1876-1882, 2006.

45. Curran S and Murray GI: Matrix metalloproteinases in tumour invasion and metastasis. J Pathol 189: 300-308, 1999.

46. Lubbe WJ, Zuzga DS, Zhou ZY, et al: Guanylyl cyclase C prevents colon cancer metastasis by regulating tumor epithelial cell matrix metalloproteinase-9. Cancer Res 69: 5472-5476, 2009.

47. Rao JS, Bhoopathi P, Chetty C, Gujrati M and Lakka SS: MMP-9 short interfering RNA induced senescence resulting in inhibition of medulloblastoma growth via p16(INK4a) and mitogen-activated protein kinase pathway. Cancer Res 67: 4956-4964, 2007.

48. Bhoopathi P, Chetty C, Kunigal S, Vanamala SK, Rao JS and Lakka SS: Blockade of tumor growth due to MMP-9 inhibition is mediated by sequential activation of $\beta 1$ integrin, ERK and NFкB. J Biol Chem 283: 1545-1552, 2008. 